\title{
Loss of Cdx2 expression in primary tumors and lymph node metastases is specific for mismatch repair-deficiency in colorectal cancer
}

\author{
Heather Dawson ${ }^{1,2}$, Viktor H. Koelzer ${ }^{1,2}$, Anne C. Lukesch ${ }^{3}$, Makhmudbek Mallaev ${ }^{3}$, Daniel Inderbitzin ${ }^{3,4}$, \\ Alessandro Lugli ${ }^{1,2}$ and Inti Zlobec ${ }^{2 *}$
}

${ }^{1}$ Department of Clinical Pathology, Institute of Pathology, University of Bern, Bern, Switzerland

${ }^{2}$ Translational Research Unit, Institute of Pathology, University of Bern, Bern, Switzerland

${ }^{3}$ Department of Visceral and Transplantation Surgery, Insel Hospital, Bern University Hospital, Bern, Switzerland

${ }^{4}$ University Clinic for Visceral Surgery and Medicine, Tiefenau Hospital, Bern, Switzerland

\section{Edited by:}

Kenichi Hirabayashi, Tokai University

School of Medicine, Japan

Reviewed by:

Shuji Ogino, Dana-Farber Cancer

Institute, USA

Hiroaki Taniguchi, The University of

Tokyo, Japan

Qianghua Xia, The Children's Hospital

of Philadelphia, USA

\section{*Correspondence:}

Inti Zlobec, Translational Research

Unit, Institute of Pathology, University

of Bern, Murtenstrasse 31, Bern

CH-3010, Switzerland

e-mail:inti.zlobec@

pathology.unibe.ch
Background: Approximately $20 \%$ of all colorectal cancers are hypothesized to arise from the "serrated pathway" characterized by mutation in BRAF, high-level $\mathrm{CpG}$ Island Methylator Phenotype, and microsatellite instability/mismatch repair (MMR)-deficiency. MMRdeficient cancers show frequent losses of $\mathrm{Cd} \times 2$, a homeodomain transcription factor. Here, we determine the predictive value of $\mathrm{Cd} \times 2$ expression for MMR-deficiency and investigate changes in expression between primary cancers and matched lymph node metastases.

Methods: Immunohistochemistry for Cdx2, Mlh1, Msh2, Msh6, and Pms2 was performed on whole tissue sections from 201 patients with primary colorectal cancer and 59 cases of matched lymph node metastases. Receiver operating characteristic curve analysis and Area under the Curve (AUC) were investigated; association of $\mathrm{Cd} 22$ with clinicopathological features and patient survival was carried out.

Results: Loss of Cdx2 expression was associated with higher tumor grade $(p=0.0002)$, advanced $\mathrm{pT}(p=0.0166)$, and perineural invasion $(p=0.0228)$. C $d \times 2$ loss was an unfavorable prognostic factor in univariate $(p=0.0145)$ and multivariate $[p=0.0427$; HR $(95 \%$ $\mathrm{Cl}): 0.58(0.34-0.98)]$ analysis. The accuracy (AUC) for discriminating MMR-proficient and deficient cancers was 87\% [OR (95\% Cl): $0.96(0.95-0.98) ; p<0.0001]$. Specificity and negative predictive value for MMR-deficiency was 99.1 and $96.3 \%$. One hundred and seventy-four patients had MMR-proficient cancers, of which 60 (34.5\%) showed Cdx2 loss. Cdx2 loss in metastases was related to MMR-deficiency $(p<0.0001)$. There was no difference in expression between primary tumors and matched metastases.

Conclusion: Loss of $\mathrm{Cdx2}$ is a sensitive and specific predictor of MMR-deficiency, but is not limited to these tumors, suggesting that events "upstream" of the development of microsatellite instability may impact $\mathrm{Cd} \times 2$ expression.

Keywords: colorectal cancer, Cdx2, mismatch repair, microsatellite instability

\section{INTRODUCTION}

Colorectal cancer is a heterogeneous disease at the clinical, histopathological, and molecular level (1). Several molecular classifications of colorectal cancer based on features such as chromosomal instability, point mutations ( $A P C, K R A S, B R A F)$, microsatellite instability (MSI), and CpG island methylation have been proposed (2-4). It is now generally accepted that approximately $20 \%$ of all colorectal cancers arise from serrated adenomas that have undergone a series of genetic changes (5). In the earliest phase of this "serrated pathway" it is hypothesized that mutational activation of $B R A F$ leads to an initial burst in proliferation within the normal colonic epithelium followed by $\mathrm{p} 16$-induced cell senescence (oncogene-activated senescence) $(6,7)$. Escape from senescence would be achieved by methylation of p16INK4A, loss of p53 function, or silencing of insulin-like growth factor binding protein 7 (IGFBP7). Responsible for this silencing is the $\mathrm{CpG}$ Island Methylator Phenotype (CIMP), a state of aberrant methylation of promoter region $\mathrm{CPG}$ islands associated with transcriptional inactivation of tumor suppressor genes (8). These changes lead to the development of sessile serrated adenomas (SSA) that may eventually progress to colorectal cancers (4).

Importantly, among the relevant tumor suppressor genes frequently silenced by CIMP is $M L H 1$, a critical gene involved in DNA mismatch repair $(9,10)$. When hypermethylated, $M L H 1$ contributes to the development of MSI, a feature observed in $15 \%$ of all cases. Defects in the DNA mismatch repair system can be observed by immunohistochemistry for microsatellite instability/mismatch repair (MMR) proteins, such as Mlh1, Msh2, Msh6, and Pms2 $(11,12)$ with negativity in any one of these proteins a sign of MMR-deficiency. 
Interestingly, some studies have observed that MMR-deficient colorectal cancers show a frequent loss of Cdx2, a tumor suppressor gene and homeodomain transcription factor that functions to regulate intestinal epithelial cell differentiation (13-15). Reduced Cdx2 expression has additionally been associated with increased migration and invasion of cancer cells and may play a role in the epithelial mesenchymal transition (EMT) by disrupting WNT pathway signaling (16-21).

The aim of this study is to determine the predictive value of Cdx2 expression for MMR-deficiency, the association with clinicopathological features and patient survival as well as to investigate changes in $\mathrm{Cdx} 2$ expression between primary cancers and matched lymph node metastases.

\section{PATIENTS AND METHODS \\ PATIENTS}

The patient cohort consisted of 201 non-consecutive patients treated at the Visceral and Transplantation Surgery department the Insel Hospital in Bern, Switzerland between 2002 and 2011. Gender and age information was available for all patients. Histopathology was systematically re-reviewed. TNM staging was performed in accordance with the seventh edition of the AJCC/UICC staging manual. Clinical metastasis staging (cM) information was available for 190 patients. Lymphatic, venous, and perineural invasion could be reviewed on a majority of cases. Information on adjuvant therapy was available for 197 patients and survival time for 93 patients. No patients received neoadjuvant therapy. Median overall survival time was 54.6 months.

\section{SPECIMEN CHARACTERISTICS}

Formalin fixed ( $10 \%$ neutral buffered formalin) paraffinembedded tumor blocks were retrieved from the Institute of Pathology, University of Bern, Switzerland. One representative tumor block of primary cancer and lymph node metastases was identified for immunohistochemistry. Ethical consent was obtained from the local ethics commission for both groups.

\section{IMMUNOHISTOCHEMISTRY}

Immunohistochemistry was carried out on whole tissue sections, cut at $4 \mu \mathrm{m}$, for all primary colorectal cancers and lymph nodes (Cdx2, Mlh1, Msh2, Msh6, and Pms2). Negative controls were tested with omission of the primary antibodies. An automated Bond III instrument was used along with the following antibodies and protocols: Cdx2, Leica-Novocastra, NCL-Cdx2, 1:200, Tris 95, $30 \mathrm{~min}$; MLH1, Leica-Novocastra, NCL-MLH1, 1:200, Tris $95^{\circ}, 30 \mathrm{~min}$; MSH2, Leica-Novocastra, NCL-MSH2, 1:200, Tris 95, 30 min; MSH6, Leica-Novocastra, MSH6-L-CE, 1:1600, Tris $95^{\circ}, 30 \mathrm{~min}$; PMS2, Leica-Novocastra NCL-L-PMS2, 1:75, Tris 95, $30 \mathrm{~min}$. For $\mathrm{Cdx} 2$ expression, the percentage of positive tumor cells was estimated. For MMR proteins, any tumor cell expression was defined as positivity for that marker. MMR-deficiency was assigned to cases showing loss of any of the four proteins. Since information on family history was unavailable, no attempt was made to further subdivide patients into Lynch syndrome or sporadic MSI.

\section{STATISTICS}

The association between Cdx2 expression as continuous variable and MMR status (proficient versus deficient) was investigated using simple logistic regression analysis. Odds ratio (OR) and $95 \%$ confidence intervals (CI) were used to determine effect size. The area under the receiver operating characteristic (ROC) curve (AUC) was used to determine the discriminatory ability of Cdx2 expression for MMR-deficiency, with values closer to 1.0 indicating a better discrimination. Cutoffs for $\mathrm{Cdx} 2$ focal and diffuse expression were also assessed by ROC curve analysis, by selecting the point on the curve giving the highest sensitivity and specificity for MMR-deficiency. For the association with age, a Wilcoxon's Test was used and to test the difference in expression between tumor and lymph node, a Wilcoxon's Signed Rank Test for matched pairs. Univariate survival analysis was performed using the log-rank and Wilcoxon's tests. Multivariable survival analysis was carried out using Cox regression analysis, with "loss" of $\mathrm{Cdx} 2$ used as a baseline. Hazard ratios and 95\% CI were used to determine the effect of Cdx2 expression on overall survival. $p$-Values $<0.05$ were considered statistically significant. All analyses were carried out using SAS V9.2 (The SAS Institute, Cary, NC, USA).

\section{RESULTS}

\section{PATIENT CHARACTERISTICS}

Patient characteristics are shown in Table 1. Whole tissue sections from 201 patients were evaluated for $\mathrm{Cdx} 2$ expression. Of these, 59 patients had available lymph node metastases that underwent Cdx2 staining as well. Representative photomicrographs are shown in Figures 1A,B.

\section{ASSOCIATION OF CdX2 IN TUMOR AND LYMPH NODES WITH CLINICOPATHOLOGICAL FEATURES}

Focal Cdx2 expression was significantly more frequent in colorectal cancers with mucinous histology $(p=0.0053)$, higher tumor grade $(p=0.0002)$, more advanced pT stage $(p=0.0166)$, with perineural invasion $(p=0.0228)$, and in those receiving adjuvant therapy $(p=0.0058)$. In addition, there was a significant and adverse effect of Cdx2 loss on patient survival $(p=0.0145$; Figure 2A). This result was maintained in multivariable analysis with $\mathrm{pT}$ and $\mathrm{pN}$ classifications $[p=0.0427$; HR (95\% CI): 0.58 (0.34-0.98)] but not when clinical metastasis staging was included in the model. Although not statistically significant, possibly due to a smaller number of patients, loss of $\mathrm{Cdx} 2$ seemed to occur more frequently in tumors with lymphatic invasion $(p=0.0809)$, and in patients with metastasis $(p=0.0887)$.

Table 2 shows the associations between lymph node expression of $\mathrm{Cdx} 2$ and clinicopathological features of the primary cancers. Indeed, only tumor location was linked to loss of Cdx2 expression, which occurs more frequently in the right-side of the colon $(p=0.0088)$. Of the 59 patients with evaluable lymph nodes, information on survival was only available in 26 . Loss of Cdx2 in lymph node metastasis was marginally associated with overall survival $(p=0.0512)$.

Evaluating the matched lymph nodes and primary colorectal cancers, average expression was $66.7 \%$ in lymph nodes and $71.0 \%$ in primary tumors. Using a matched pairs analysis, this difference was not significant $(p=0.5801)$. 
Table 1 | Patient characteristics $(n=201)$ and association with $\mathrm{Cdx} 2$ expression in tumor.

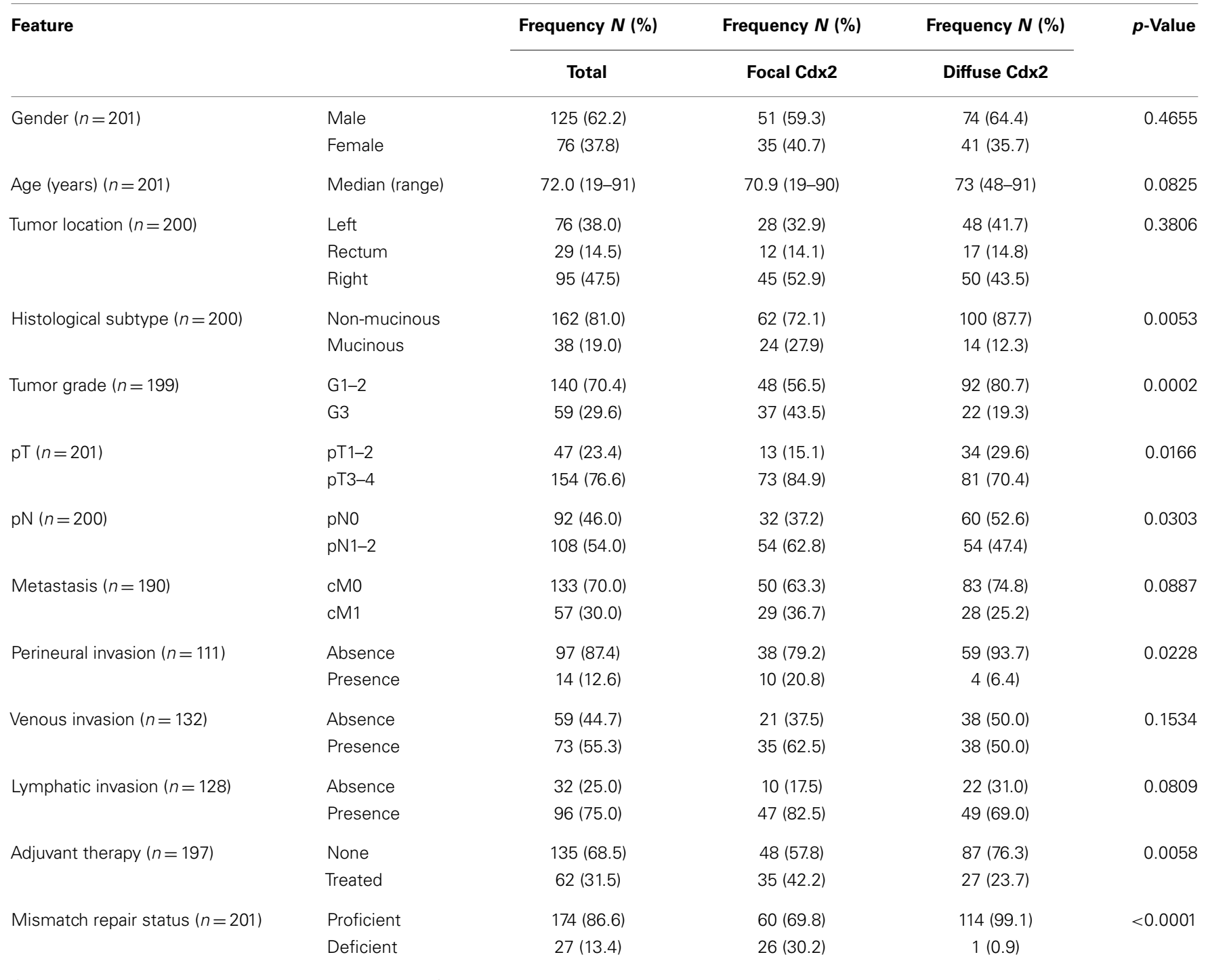

Overall survival $(n=93)$
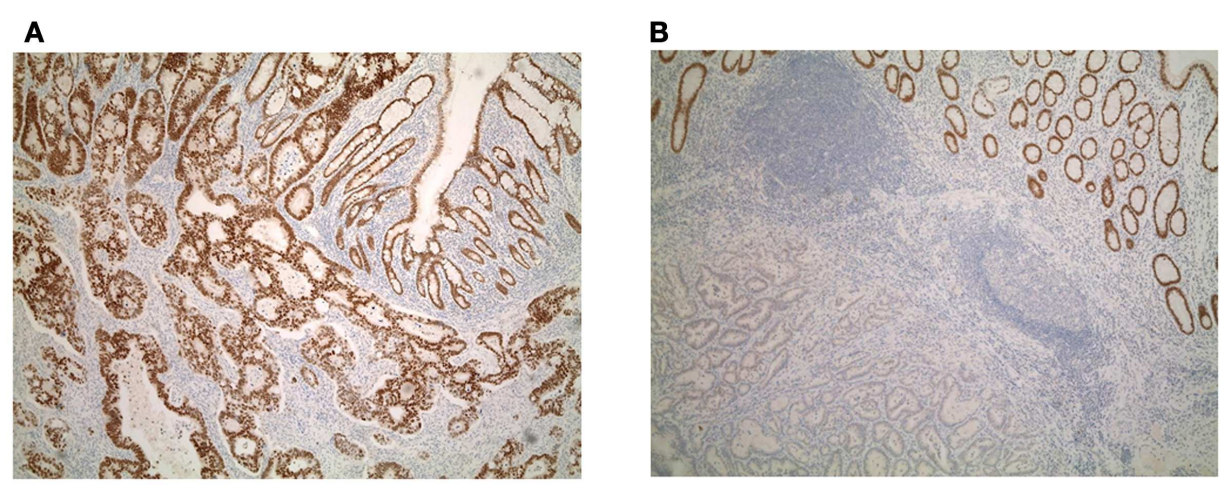

FIGURE 1 | (A) Diffuse and (B) focal expression of Cdx2 in colorectal cancers. 

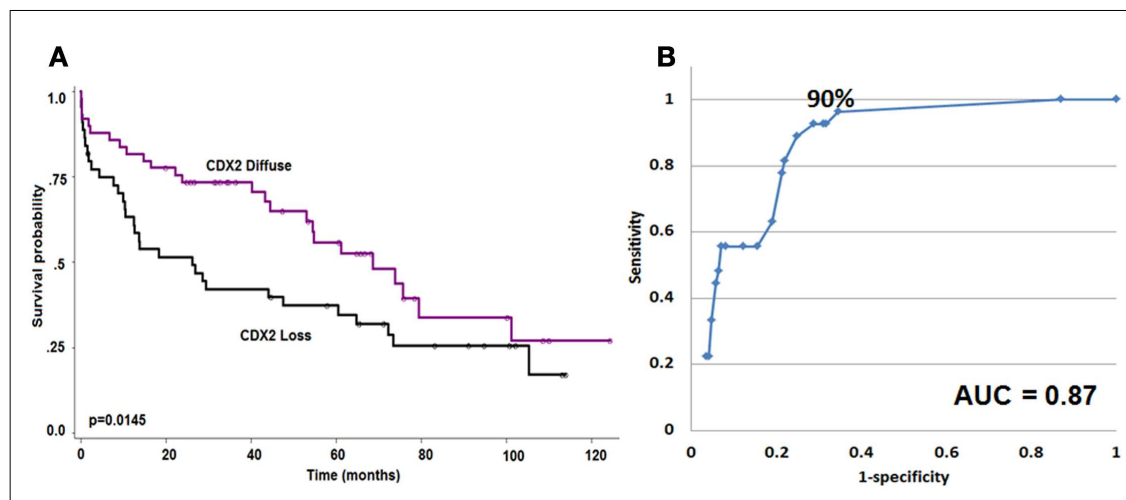

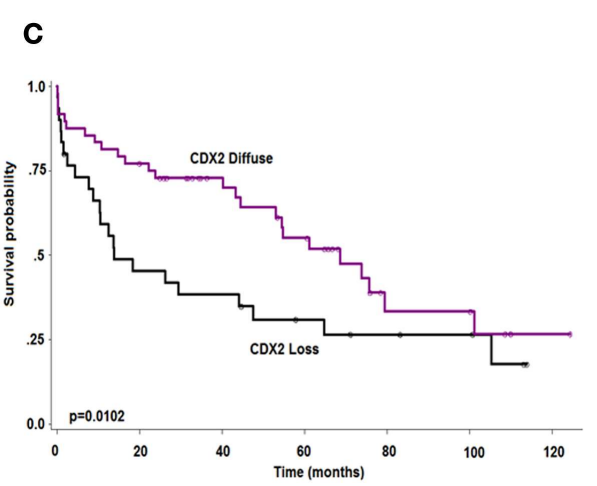

FIGURE 2 | (A) Kaplan-Meier survival curve showing effect of Cdx2 expression on overall survival $(n=93)$. (B) ROC curve highlighting the strong predictive effect and specificity of loss of $\mathrm{Cdx} 2$ expression for
MMR-deficiency. (C) Kaplan-Meier survival curve showing effect of Cdx2 expression on overall survival in MMR-proficient colorectal cancer patients only $(n=78)$. Wilcoxon's test.

Table 2 | Association of Cdx2 loss in tumor and lymph nodes with clinicopathological features ( $n=59)$.

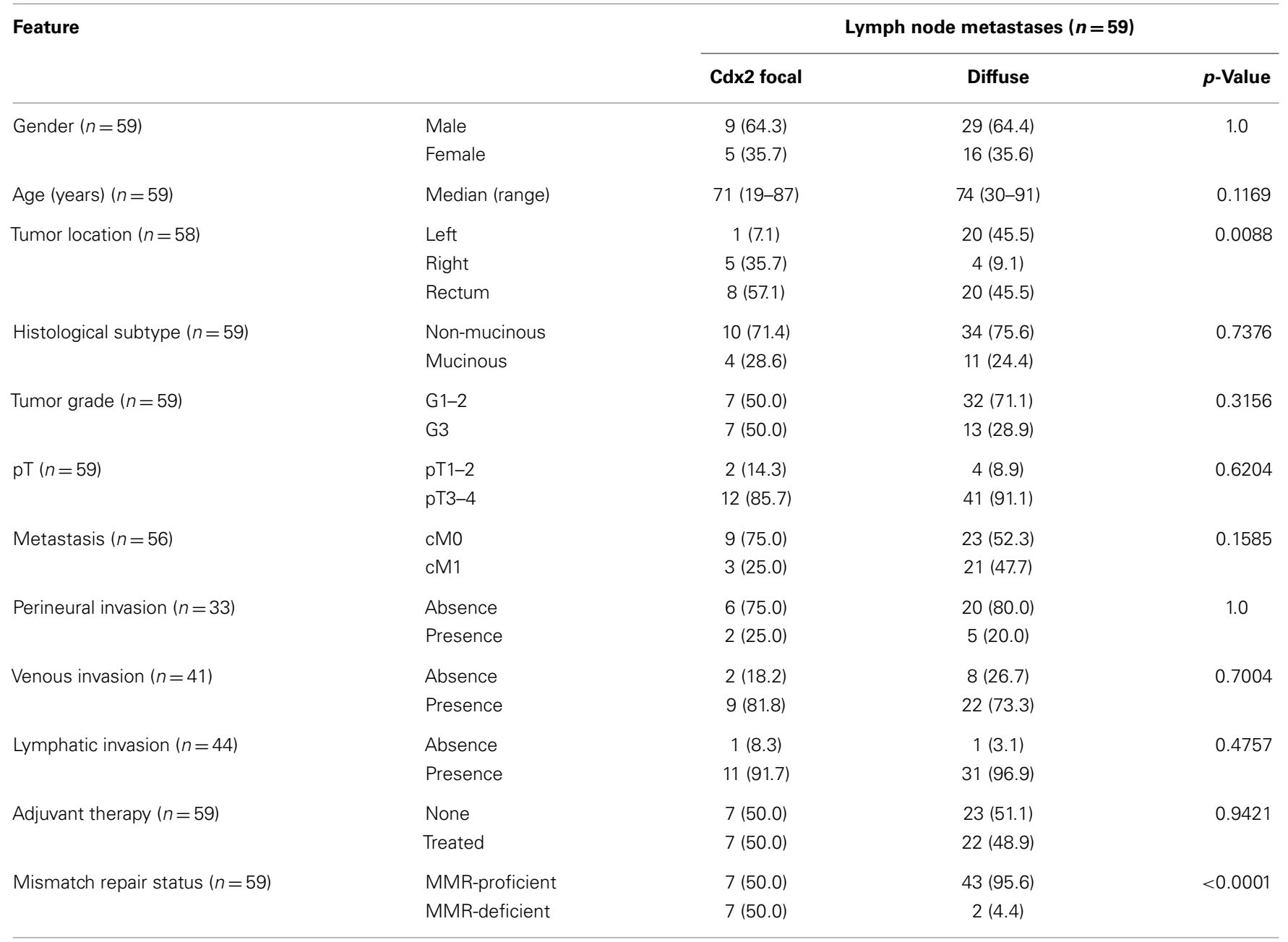

\section{Cdx2 EXPRESSION AND MISMATCH REPAIR STATUS}

There was a major significant association between reduced Cdx2 expression and MMR-deficiency. The AUC value for Cdx2 expression in tumor was 0.87 indicating $87 \%$ accuracy for discriminating MMR-proficient and - deficient cancers (Figure 2B). The OR (95\% CI) was $0.96(0.95-0.98) ; p<0.0001$. The ROC 
curve was used as a basis for the identification of an optimal threshold value for considering tumors with "focal" and "diffuse" expression and determined to be $90 \%$. Of the 115 patients with diffuse expression of Cdx2, 114 were MMR-proficient (99.1\% specificity) and of the 27 MMR-deficient patients 26 had only focal expression (96.3\% negative predictive value). There were 174 patients with MMR-proficient cancers, of which $60(34.5 \%)$ indeed showed loss of Cdx2. Cdx2 loss among patients with MMRproficient cancers was significantly and unfavorably related to survival ( $p=0.0102$; Figure 2C). Again, in multivariable analysis, $\mathrm{Cdx} 2$ loss was associated with worse outcome after adjusting for $\mathrm{pT}$ and $\mathrm{pN}[p=0.0414$; HR (95\% CI): $0.54(0.3-0.98)]$, but not when clinical metastasis stage was added.

The AUC for Cdx2 expression in lymph nodes and MMR status was 0.943 indicating $94 \%$ discriminatory ability of the protein. The OR (95\% CI) was $0.93(0.87-0.99) ; p=0.037$. Using the ROC curve for the selection of a threshold value, tumors with $<30 \%$ staining were considered "focal" and $>30 \%$ considered "diffuse" for Cdx2 expression. Of the 45 cases with diffusely expressing Cdx2, 43 were MMR-proficient (95.6\% specificity), whereas $7 / 9$ MMR-deficient cancers showed focal expression of Cdx2 (77.8\% NPV).

\section{DISCUSSION}

The findings of this study suggest that reduced expression of Cdx2 in primary tumors and lymph node metastases is an accurate predictor of MMR-deficiency in colorectal cancer. Moreover, loss of $\mathrm{Cdx} 2$ is a poor prognostic factor, even among patients with MMR-proficient cancers.

In a first step, we examined the specificity of Cdx2 for MMR status. The ROC curve for this analysis underlines the major discriminatory power of reduced $\mathrm{Cdx} 2$ expression for MMR-deficiency in both colorectal cancers and lymph nodes. Previous reports by our group and others have highlighted similar findings. Using a tissue microarray containing more than 600 patient tissues, Baba and colleagues showed a high specificity of reduced $\mathrm{Cdx} 2$ expression for MSI-high colorectal cancers (22). The protein expression of Cdx2 in MMR-proficient versus deficient cancers has been reported at 84 versus $61 \%$ on average, again using tissue microarrays (14). Our study goes one step further and uses whole tissue sections for the establishment of both MMR status and Cdx2 expression. Indeed, all MMR-deficient cancers with the exception of one case showed only focal positivity for $\mathrm{Cdx} 2$ expression.

Despite this observation, a subgroup of MMR-proficient cancers also shows focal positivity for Cdx2. Our hypothesis is that $\mathrm{Cdx} 2$ loss may be an important marker of other molecular changes associated with the serrated pathway to colorectal cancer, including $B R A F$ mutation and high-level CIMP. Indeed, we could previously show using a cohort of more than 300 patients, that loss of Cdx2 was nearly $100 \%$ specific for $B R A F$ mutation, and found in 23/24 mutated cases (23). Baba and colleagues as well as Walsh et al. found loss of $\mathrm{Cdx} 2$ in $B R A F$ mutated tumors and a significantly more frequent number of cases in tumors with CIMP-H $(22,24)$. Loss of $\mathrm{Cdx} 2$ has also been found to be an independent predictor of the CIMP-H phenotype (25). Figure 3 illustrates some of the changes hypothesized to occur during the serrated pathway. We believe that loss of $\mathrm{Cdx} 2$ expression occurs prior

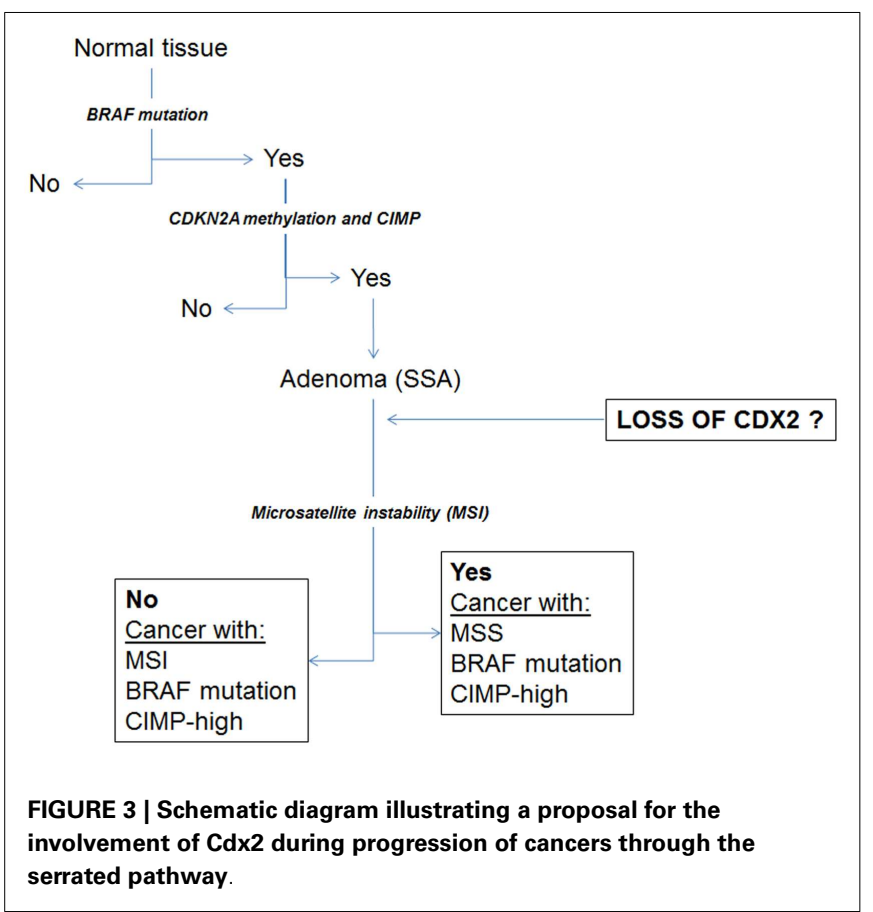

to the establishment of MSI and only after the development of both $B R A F$ mutation and CIMP. Although the evidence fits well for an involvement of $\mathrm{Cdx} 2$ in the serrated pathway, whether this molecule is actually functionally involved as a cause rather than a consequence of progression of tumors within this pathway has not yet been established.

Next, we evaluated the association between focal expression of $\mathrm{Cdx} 2$ and clinicopathological features. Our results point toward an association of Cdx2 with an array of important and adverse prognostic features including unfavorable overall survival. Our findings are in line with previous work from our group using a single punch tissue microarray showing strong correlations between $\mathrm{Cdx} 2$ loss and pT, pN, tumor grade, and vascular invasion on more than 1000 tumors. Baba and colleagues showed similar results of Cdx2 loss with more advanced TNM stage, higher tumor grade, mucinous, or signet ring cell histology (22). These results are in agreement with Choi et al. who show loss of Cdx2 expression associated with advanced Dukes' stage and more poorly differentiated cancers (26). Unfavorable survival times are reported by several groups upon reduced $\mathrm{Cdx} 2$ expression $(22,27)$. In addition, the predilection for female gender and more right-sided tumor location has also been observed in other studies $(22,28)$. We also show that the unfavorable impact of $\mathrm{Cdx} 2$ is maintained in patients with MMR-deficient cancers.

Thirdly, we evaluated for the first time Cdx2 expression in matched lymph node metastases. We found no differences in expression between lymph nodes and primary colorectal cancers. These results appear to indicate that a further "evolution" leading to loss of Cdx2 after lymph node spread is unlikely.

To conclude, Cdx2 is significantly reduced in patients with MMR-deficient colorectal cancers, but is not limited to these tumors. It is an unfavorable prognostic factor, even among 
patients with MMR-proficient cancers. Taken together with previous reports on $B R A F$ and CIMP, we hypothesize that Cdx2 loss may play an early role in the progression of cancers arising through the serrated pathway.

\section{REFERENCES}

1. Jass JR. Molecular heterogeneity of colorectal cancer: implications for cancer control. Surg Oncol (2007) 16(Suppl 1):S7-9. doi:10. 1016/j.suronc.2007.10.039

2. Domingo E, Ramamoorthy R, Oukrif D, Rosmarin D, Presz M, Wang $\mathrm{H}$, et al. Use of multivariate analysis to suggest a new molecular classification of colorectal cancer. J Pathol (2013) 229:441-8. doi: 10.1002/path.4139

3. Jass JR. Classification of colorectal cancer based on correlation of clinical, morphological and molecular features. Histopathology (2007) 50:113-30. doi:10.1111/j. 1365-2559.2006.02549.x

4. Leggett B, Whitehall V. Role of the serrated pathway in colorectal cancer pathogenesis. Gastroenterology (2010) 138:2088-100. doi:10.1053/ j.gastro.2009.12.066

5. Hawkins NJ, Ward RL. Sporadic colorectal cancers with microsatellite instability and their possible origin in hyperplastic polyps and serrated adenomas. J Natl Cancer Inst (2001) 93:1307-13. doi:10.1093/jnci/93.17. 1307

6. Carragher LA, Snell KR, Giblett SM, Aldridge VS, Patel B, Cook SJ, et al. V600EBraf induces gastrointestinal crypt senescence and promotes tumour progression through enhanced CpG methylation of p16INK4a. EMBO Mol Med (2010) 2:458-71. doi:10.1002/emmm.201000099

7. Kriegl L, Neumann J, Vieth M, Greten FR, Reu S, Jung A, et al. Up and downregulation of p16(Ink4a) expression in BRAFmutated polyps/adenomas indicates a senescence barrier in the serrated route to colon cancer. Mod Pathol (2011) 24:1015-22. doi:10. 1038/modpathol.2011.43

8. Toyota M, Ahuja N, Ohe-Toyota M, Herman JG, Baylin SB, Issa JP. CpG island methylator phenotype in colorectal cancer. Proc Natl Acad Sci U S A (1999) 96:8681-6. doi:10.1073/pnas.96.15. 8681

9. Hawkins N, Norrie M, Cheong K, Mokany E, Ku SL, Meagher $\mathrm{A}$, et al. CpG island methylation in sporadic colorectal cancers and its relationship to microsatellite instability. Gastroenterology (2002) 122:1376-87. doi:10.1053/gast.2002.32997

10. Jass JR, Young J, Leggett BA. Hyperplastic polyps and DNA microsatellite unstable cancers of the colorectum. Histopathology (2000) 37:295-301. doi:10.1046/j. 1365-2559.2000.01028.x

11. Halvarsson B, Lindblom A, Rambech E, Lagerstedt K, Nilbert M. The added value of PMS2 immunostaining in the diagnosis of hereditary nonpolyposis colorectal cancer. Fam Cancer (2006) 5:353-8. doi:10.1007/s10689-006-0005-9

12. Pedroni M, Sala E, Scarselli A, Borghi F, Menigatti M, Benatti P, et al. Microsatellite instability and mismatch-repair protein expression in hereditary and sporadic colorectal carcinogenesis. Cancer Res (2001) 61:896-9.

13. De Lott LB, Morrison C, Suster S, Cohn DE, Frankel WL. CDX2 is a useful marker of intestinal-type differentiation: a tissue microarraybased study of 629 tumors from various sites. Arch Pathol Lab Med (2005) 129:1100-5.

14. Lugli A, Tzankov A, Zlobec I, Terracciano LM. Differential diagnostic and functional role of the multi-marker phenotype CDX2/CK20/CK7 in colorectal cancer stratified by mismatch repair status. Mod Pathol (2008) 21:1403-12. doi:10.1038/modpathol.2008.117

15. Werling RW, Yaziji H, Bacchi CE, Gown AM. CDX2, a highly sensitive and specific marker of adenocarcinomas of intestinal origin: an immunohistochemical survey of 476 primary and metastatic carcinomas. Am J Surg Pathol (2003) 27:303-10. doi:10.1097/00000478200303000-00003

16. Brabletz T, Spaderna S, Kolb J, Hlubek F, Faller G, Bruns CJ, et al. Down-regulation of the homeodomain factor $\mathrm{Cdx} 2$ in colorectal cancer by collagen type I: an active role for the tumor environment in malignant tumor progression. Cancer Res (2004) 64:6973-7. doi:10.1158/0008-5472. CAN-04-1132

17. Funakoshi S, Kong J, Crissey MA, Dang L, Dang D, Lynch JP. Intestine-specific transcription factor $\mathrm{Cdx} 2$ induces E-cadherin

\section{ACKNOWLEDGMENTS}

This study was partially funded by the Bernese Cancer League. We thank Mary Economou and Caroline Hammer for excellent technical support.

function by enhancing the trafficking of E-cadherin to the cell membrane. Am J Physiol Gastrointest Liver Physiol (2010) 299:G1054-67. doi:10.1152/ajpgi.00297.2010

18. Gross I, Duluc I, Benameur T, Calon A, Martin E, Brabletz T, et al. The intestine-specific homeobox gene Cdx2 decreases mobility and antagonizes dissemination of colon cancer cells. Oncogene (2008) 27:107-15. doi:10.1038/sj. onc. 1210601

19. Guo RJ, Funakoshi S, Lee HH, Kong J, Lynch JP. The intestine-specific transcription factor $\mathrm{Cdx} 2$ inhibits beta-catenin/TCF transcriptional activity by disrupting the betacatenin-TCF protein complex. Carcinogenesis (2010) 31:159-66. doi:10.1093/carcin/bgp213

20. Hinkel I, Duluc I, Martin E, Guenot D, Freund JN, Gross I. Cdx2 controls expression of the protocadherin Mucdhl, an inhibitor of growth and beta-catenin activity in colon cancer cells. Gastroenterol ogy (2012) 142(875-85):e873. doi: 10.1053/j.gastro.2011.12.037

21. Zheng JB, Sun XJ, Qi J, Li SS, Wang W, Ren HL, et al. Effects of homeodomain protein CDX2 expression on the proliferation and migration of lovo colon cancer cells. Pathol Oncol Res (2011) 17:743-51. doi:10. 1007/s12253-011-9380-0

22. Baba Y, Nosho K, Shima K, Freed E, Irahara N, Philips J, et al. Relationship of CDX2 loss with molecular features and prognosis in colorectal cancer. Clin Cancer Res (2009) 15:4665-73. doi:10. 1158/1078-0432.CCR-09-0401

23. Zlobec I, Bihl MP, Schwarb H, Terracciano L, Lugli A. Clinicopathological and protein characterization of BRAF- and K-RAS-mutated colorectal cancer and implications for prognosis. Int J Cancer (2010) 127:367-80. doi:10.1002/ijc.25042

24. Walsh MD, Clendenning $M$, Williamson E, Pearson SA, Walters RJ, Nagler B, et al. Expression of MUC2, MUC5AC, MUC5B, and MUC6 mucins in colorectal cancers and their association with the $\mathrm{CpG}$ island methylator phenotype. Mod Pathol (2013). doi:10.1038/modpathol.2013.101

25. Zlobec I, Bihl M, Foerster A, Rufle A, Lugli A. Comprehensive analysis of
CpG island methylator phenotype (CIMP)-high, -low, and -negative colorectal cancers based on protein marker expression and molecular features. J Pathol (2011) 225:336-43. doi:10.1002/path.2879

26. Choi BJ, Kim CJ, Cho YG, Song JH, Kim SY, Nam SW, et al. Altered expression of CDX2 in colorectal cancers. APMIS (2006) 114:50-4. doi:10.1111/j.1600-0463. 2006.apm_292.x

27. Hong KD, Lee D, Lee Y, Lee SI, Moon HY. Reduced CDX2 expression predicts poor overall survival in patients with colorectal cancer. Am Surg (2013) 79:353-60.

28. Minoo P, Zlobec I, Peterson M, Terracciano L, Lugli A. Characterization of rectal, proximal and distal colon cancers based on clinicopathological, molecular and protein profiles. Int $\mathrm{J}$ Oncol (2010) 37:707-18. doi:10.3892/ijo_ 00000720

Conflict of Interest Statement: The authors declare that the research was conducted in the absence of any commercial or financial relationships that could be construed as a potential conflict of interest.

Received: 28 July 2013; accepted: 26 September 2013; published online: 11 October 2013.

Citation: Dawson $H$, Koelzer VH, Lukesch AC, Mallaev M, Inderbitzin D, Lugli A and Zlobec I (2013) Loss of Cdx2 expression in primary tumors and lymph node metastases is specific for mismatch repair-deficiency in colorectal cancer. Front. Oncol. 3:265. doi: 10.3389/fonc. 2013.00265

This article was submitted to Gastrointestinal Cancers, a section of the journal Frontiers in Oncology.

Copyright (C) 2013 Dawson, Koelzer, Lukesch, Mallaev, Inderbitzin, Lugli and Zlobec. This is an open-access article distributed under the terms of the Creative Commons Attribution License (CC BY). The use, distribution or reproduction in other forums is permitted, provided the original author(s) or licensor are credited and that the original publication in this journal is cited, in accordance with accepted academic practice. No use, distribution or reproduction is permitted which does not comply with these terms. 